\title{
A MODIFIED ASYMPTOTIC METHOD FOR A VAN-DER-POL OSCILLATOR WITH LARGE CUBIC RESTORING NONLINEARITY
}

\author{
NGUYEN VAN DiNH \\ Institute of Mechanics
}

\begin{abstract}
A modified asymptotic method is proposed and applied to evaluate the frequency of self-excited oscillation in a Van-Der-Pol oscillator with large cubic restoring nonlinearity. The result obtained can be used for large enough nonlinearity.
\end{abstract}

\section{Introduction}

In [3], to evaluate the frequency of steady self-excited oscillation in a Van-Der-Pol oscillator with large cubic restoring nonlinearity, a modified Poincaré method has been presented. There, assuming that the strongly nonlinear oscillator of interest is near certain linear one with unknown (to be evaluated) frequency, a formal small parameter is introduced and the governing differential equation is written in the form corresponding to weakly nonlinear system.

In this article, based on the same assumption and also on the variant of the asymptotic procedure presented in $[1,2]$, a modified asymptotic method is proposed to examine the problem considered. The results obtained are identical with those given in [3]. Compared with the modified Poincaré method, the modified asymptotic method is a little more complicated. This results from the fact that in the asymptotic method, we have to establish not only the algebraic equations determining steady state but the differential equations governing the amplitude-phase variation of the "general" solution. However, as compensation, the stability study is rather simple.

\section{Systems under consideration. Frequency from the usual asymptotic method}

Consider a Van-Der-Pol oscillator described by the differential equation

$$
\ddot{x}+x=-\gamma x^{3}+h\left(1-x^{2}\right) \dot{x}
$$

where the significations of all the notations have been explained in [3]; $h>0$ is assumed to be small but $\gamma>0$ may be large enough.

The problem posed is to evaluate the frequency of steady self-excited oscillations satisfying the initial condition

$$
\dot{x}(0)=0 \text {. }
$$

For the sake of comparison, the usual asymptotic method (with a modified procedure) as presented in [2] is first used to discuss the case of small $\gamma$. 
Being small, $\gamma$ as $h$ can be labeled by a small formal parameter $\varepsilon$. So, the system under consideration belongs to a weakly nonlinear type and its governing differential equation has the familiar form:

$$
\ddot{x}+x=\varepsilon\left\{-\gamma x^{3}+h\left(1-x^{2}\right) \dot{x}\right\} .
$$

Following asymptotic expansions are used:

$$
\begin{aligned}
x & =a \cos \psi+\varepsilon u_{1}(a, \psi)+\varepsilon^{2} u_{2}(a, \psi)+\ldots \\
\dot{a} & =\varepsilon A_{1}(a)+\varepsilon^{2} A_{2}(a)+\ldots \\
\dot{\psi} & =1+\varepsilon B_{1}(a)+\varepsilon^{2} B_{2}(a)+\ldots
\end{aligned}
$$

where $a$ is the amplitude of order $\varepsilon^{0}$ of the first harmonic; $\psi$ is the full phase angle with initial value $\psi(0)=0$ (since the system considered is autonomous); $A_{i}, B_{i}(i=1,2, \ldots)$ are functions of $a$ and $u_{i}(i=1,2, \ldots)$ are functions of $(a, \psi), 2 \pi$-periodic with respect to $\psi$. Note that $u_{i}$ may contain the first harmonics $\sin \psi$ and $\cos \psi$.

Steady self-excited oscillation with $a_{0}$ as amplitude of order $\varepsilon^{0}$ is determined such that in each step of approximation (not at the end) following conditions are satisfied:

1 -

$$
A_{i}\left(a_{0}\right)=0 \text { for every } i
$$

$2-$

$$
\frac{\partial u_{i}\left(a_{0}, 0\right)}{\partial \psi}=0 \text { for every } i
$$

It is not difficult to recognize that $(1.7),(1.8)$ represent the stationarity condition and the initial condition respectively.

In the first approximation, we have:

$$
\begin{aligned}
& -2 A_{1} \sin \psi-2 a B_{1} \cos \psi+\frac{\partial^{2} u_{1}}{\partial \psi^{2}}+u_{1}=-\gamma a^{3} \cos ^{3} \psi-h a\left(1-a^{2} \cos ^{2} \psi\right) \sin \psi \\
& =-h a\left(1-\frac{a^{2}}{4}\right) \sin \psi-\frac{3 \gamma}{4} a^{3} \cos \psi-\frac{\gamma a^{3}}{4} \cos 3 \psi+\frac{h a^{3}}{4} \sin 3 \psi .
\end{aligned}
$$

Hence:

$$
\begin{aligned}
& A_{1}(a)=\frac{h a}{2}\left(1-\frac{a^{2}}{4}\right), \quad B_{1}=\frac{3 \gamma}{8} a^{2}, \\
& \frac{\partial^{2} u_{1}}{\partial \psi^{2}}+u_{1}=-\frac{\gamma a^{3}}{4} \cos 3 \psi+\frac{h a^{3}}{4} \sin 3 \psi, \\
& u_{1}=\frac{\gamma a^{3}}{32} \cos 3 \psi-\frac{h a^{3}}{32} \sin 3 \psi+a_{1} \cos \psi+b_{1} \sin \psi,
\end{aligned}
$$

where $a_{1}, b_{1}$ are two constants to be chosen.

By $a_{0}$ we denote the amplitude of order $\varepsilon^{0}$ of the self-excited oscillation; it is determined by the equation:

$$
A_{1}(a)=0 \quad \text { or } \quad a_{0}=2 .
$$

Using (1.12), the additional frequency $\varepsilon B_{1}(a)$ of order $\varepsilon$ is:

$$
B_{1}\left(a_{0}\right)=\frac{3 \gamma a_{0}^{2}}{8}=\frac{3 \gamma}{2} .
$$


The initial condition (1.8) for $i=1$ gives:

$$
\frac{\partial u_{1}\left(a_{0}, 0\right)}{\partial \psi}=-\frac{3 h a_{0}^{3}}{32}+b_{1}=0 \quad \text { or } \quad b_{1}=b_{10}=\frac{3 h}{4} .
$$

In the second approximation, we have:

$$
\begin{aligned}
& -2 A_{2} \sin \psi-2 a B_{2} \cos \psi+\frac{\partial^{2} u_{2}}{\partial \psi^{2}}+u_{2}= \\
& =-A_{1} \frac{\partial A_{1}}{\partial a} \cos \psi+2 A_{1} B_{1} \sin \psi+a A_{1} \frac{\partial B_{1}}{\partial a} \sin \psi+a B_{1}^{2} \cos \psi-2 A_{1} \frac{\partial^{2} u_{1}}{\partial \psi \partial a} \\
& -2 B_{1} \frac{\partial^{2} u_{1}}{\partial \psi^{2}}-3 \gamma a^{2} u_{1} \cos ^{2} \psi+h a^{2} u_{1} \sin 2 \psi \\
& +h\left(1-a^{2} \cos ^{2} \psi\right)\left(A_{1} \cos \psi-a B_{1} \sin \psi+\frac{\partial u_{1}}{\partial \psi}\right) .
\end{aligned}
$$

Hence:

$$
\begin{aligned}
& A_{2}(a)=\frac{-1}{2}\left\{\frac{3 \gamma a^{2}}{2} A_{1}-h\left(1-\frac{3 a^{2}}{4}\right) a_{1}-\frac{3 \gamma h a^{3}}{8}+\frac{\gamma h a^{5}}{8}\right\} \\
& B_{2}(a)=\frac{-1}{2 a}\left\{\left[h\left(1-\frac{3 a^{2}}{4}\right)-\frac{\partial A_{1}}{\partial a}\right] A_{1}-\frac{3 \gamma a^{2}}{2} a_{1}+h\left(1-\frac{a^{2}}{4}\right) b_{1}+\frac{h^{2} a^{5}}{128}+\frac{15 \gamma^{2} a^{5}}{128}\right\} .
\end{aligned}
$$

The stationarity condition (1.7) for $i=2$ leads to the equations:

$$
A_{2}\left(a_{0}\right)=0 \quad \text { or } \quad 2 h a_{1}-3 \gamma h+\dot{4} \gamma h=0 \quad \text { i.e. } \quad a_{1}=a_{10}=\frac{-\gamma}{2} .
$$

Using (1.12), (1.14), (1.18), the additional frequency of order $\varepsilon^{2}$ is obtained:

$$
B_{2}\left(a_{0}\right)=-\frac{27 \gamma^{2}+h^{2}}{16}
$$

Thus, the first two formulas for frequency are:

$$
\begin{aligned}
\omega_{I} & =1+\varepsilon B_{1}\left(a_{0}\right)=1+\varepsilon \frac{3 \gamma}{2}, \\
\omega_{I I} & =1+\varepsilon B_{1}\left(a_{0}\right)+\varepsilon^{2} B_{2}\left(a_{0}\right)=1+\varepsilon \frac{3 \gamma}{2}-\varepsilon^{2} \frac{27 \gamma^{2}+h^{2}}{16} .
\end{aligned}
$$

In the second approximation the stability condition is

$$
\left(\varepsilon \frac{\partial A_{1}}{\partial a}+\varepsilon^{2} \frac{\partial A_{2}}{\partial a}\right)_{0}<0 .
$$




\section{$3 \quad$ Frequency from a modified asymptotic method}

In this section, a modified asymptotic method is proposed to determine steady state for the case of large cubic nonlinearity $\gamma$. As in [3], assuming that in the domain $(x, \dot{x})$ considered, the system of interest should be near certain linear one with unknown frequency $\omega$, we rewrite (1.1) as:

$$
\ddot{x}+\omega^{2} x=\mu\left\{\left(\omega^{2}-1\right) x-\gamma x^{3}+h\left(1-x^{2}\right) \dot{x}\right\},
$$

where $\mu$ is the new small formal parameter introduced to indicate the smallness (by assumption) of the right hand side (especially, the smallness of the sum $\left.\left(\omega^{2}-1\right) x-\gamma x^{3}\right)$.

The asymptotic expansions are now of the form:

$$
\begin{aligned}
x & =a \cos \psi+\mu u_{1}(a, \psi)+\mu^{2} u_{2}(a, \psi)+\ldots, \\
\dot{a} & =\mu A_{1}(a)+\mu^{2} A_{2}(a)+\ldots, \\
\omega & =\dot{\psi}=\omega_{0}+\mu B_{1}(a)+\mu^{2} B_{2}(a)+\ldots,
\end{aligned}
$$

where $\omega_{0}$ (not 1 as in the case of small $\gamma$ ) is the approximate frequency of order $\mu^{0}$.

It is not difficult to establish following expressions:

$$
\begin{aligned}
\omega^{2} x= & \omega_{0}^{2} a \cos \psi+\mu\left\{\omega_{0}^{2} u_{1}+2 \omega_{0} a B_{1} \cos \psi\right\} \\
& +\mu^{2}\left\{\omega_{0}^{2} u_{2}+2 \omega_{0} u_{1} B_{1}+\left(2 \omega_{0} B_{2}+B_{1}^{2}\right) a \cos \psi\right\}+\ldots \\
\dot{x}= & -\omega_{0} a \sin \psi+\mu\left\{A_{1} \cos \psi-a B_{1} \sin \psi+\omega_{0} \frac{\partial u_{1}}{\partial \psi}\right\} \\
& +\mu^{2}\left\{A_{2} \cos \psi-a B_{2} \sin \psi+A_{1} \frac{\partial u_{1}}{\partial a}+B_{1} \frac{\partial u_{1}}{\partial \psi}+\omega_{0} \frac{\partial u_{2}}{\partial \psi}\right\}+\ldots, \\
\ddot{x}= & -\omega_{0}^{2} \cos \psi+\mu\left\{-2 \omega_{0} A_{1} \sin \psi-2 a \omega_{0} B_{1} \cos \psi+\omega_{0}^{2} \frac{\partial^{2} u_{1}}{\partial \psi^{2}}\right\} \\
& +\mu^{2}\left\{-2 \omega_{0} A_{2} \sin \psi-2 a \omega_{0} B_{2} \cos \psi+A_{1} \frac{\partial A_{1}}{\partial a} \cos \psi\right. \\
& -2 A_{1} B_{1} \sin \psi-a A_{1} \frac{\partial B_{1}}{\partial a} \sin \psi-a B_{1}^{2} \cos \psi \\
& \left.+2 \omega_{0} A_{1} \frac{\partial^{2} u_{1}}{\partial \psi^{2}}+2 \omega_{0} B_{2} \frac{\partial^{2} u_{1}}{\partial \psi^{2}}+\omega_{0}^{2} \frac{\partial^{2} u_{2}}{\partial \psi^{2}}\right\}
\end{aligned}
$$

Substituting (2.2), (2.4), (2.5) into (2.1), then equating the terms of like powers of $\mu$ yield:

In the first approximation:

$$
\begin{aligned}
& -2 \omega_{0} A_{1} \sin \psi+\omega_{0}^{2}\left(\frac{\partial^{2} u_{1}}{\partial \psi^{2}}+u_{1}\right) \\
& =\left(\omega_{0}^{2}-1\right) a \cos \psi-\gamma a^{3} \cos ^{3} \psi-h\left(1-a^{2} \cos ^{2} \psi\right) \omega_{0} a \sin \psi \\
& =-h \omega_{0} a\left(1-\frac{a^{2}}{4}\right) \sin \psi+\left(\omega_{0}^{2}-1-\frac{3 \gamma}{4} a^{2}\right) a \cos \psi+\frac{h \omega_{0} a^{3}}{4} \sin 3 \psi-\frac{\gamma a^{3}}{4} \cos 3 \psi .
\end{aligned}
$$

Equating the terms of like harmonics in both sides of (2.6) we obtain:

$$
\begin{aligned}
& -2 \omega_{0} A_{1}=-h \omega_{0} a\left(1-\frac{a^{2}}{4}\right), \quad \omega_{0}^{2}=1+\frac{3 \gamma}{4} a^{2}, \\
& \omega_{0}^{2}\left(\frac{\partial^{2} u_{1}}{\partial \psi^{2}}+u_{1}\right)=-\frac{\gamma a^{3}}{4} \cos 3 \psi+\frac{h \omega_{0} a^{3}}{4} \sin 3 \psi .
\end{aligned}
$$


The amplitude $a_{0}$ of order $\mu^{0}$ of the steady self-excited oscillation is determined by the stationarity condition.

$$
A_{1}(a)=0 \quad \text { or } \quad a_{0}=2 .
$$

Using (2.9), from the second relation of (2.7), we obtain:

$$
\omega_{0}^{2}=1+\frac{3 \gamma}{4} a_{0}^{2}=1+3 \gamma
$$

The expression of $u_{1}$ is:

$$
u_{1}=\frac{\gamma a^{3}}{32 \omega_{0}^{2}} \cos 3 \psi-\frac{h \omega_{0} a^{3}}{32 \omega_{0}^{2}} \sin 3 \psi+a_{1} \cos \psi+b_{1} \sin \psi
$$

where $a_{1}, b_{1}$ are two constants to be chosen.

To satisfy the initial condition (1.8) for $i=1$, the constant $b_{1}$ should be taken so that:

$$
\frac{\partial u_{1}\left(a_{0}, 0\right)}{\partial \psi}=0 \quad \text { or } \quad b_{1}=b_{10}=\frac{3 h \omega_{0} a_{0}^{3}}{32 \omega_{0}^{2}}=\frac{3 h}{4 \sqrt{1+3 \gamma}} .
$$

Note that in the first approximation, $B_{1}$ is absent and $a_{1}$ remains indeterminate.

In the second approximation, we have:

$$
\begin{aligned}
& -2 \omega_{0} A_{2} \sin \psi+\omega_{0}^{2}\left(\frac{\partial^{2} u_{2}}{\partial \psi^{2}}+u_{2}\right)= \\
& =-A_{1} \frac{\partial A_{1}}{\partial a} \cos \psi+2 A_{1} B_{1} \sin \psi+a \frac{\partial B_{1}}{\partial a} A_{1} \sin \psi-2 \omega_{0} A_{1} \frac{\partial^{2} u_{1}}{\partial \psi \partial a} \\
& -2 \omega_{0} B_{1}\left(\frac{\partial^{2} u_{1}}{\partial \psi^{2}}+u_{1}\right)+\left(\omega_{0}^{2}-1\right) a_{1}+2 \omega_{0} a B_{1} \cos \psi-3 \gamma a^{2} u_{1} \cos ^{2} \psi \\
& +h \omega_{0} a^{2} \sin 2 \psi \cdot u_{1}+\left(A_{1} \cos \psi-a B_{1} \sin \psi+\omega_{0} \frac{\partial u_{1}}{\partial \psi}\right) h\left(1-a^{2} \cos ^{2} \psi\right) .
\end{aligned}
$$

Hence:

$$
\begin{aligned}
-2 \omega_{0} A_{2}= & 2 A_{1} B_{1}+a A_{1} \frac{\partial B_{1}}{\partial a}-h\left(1-\frac{a^{2}}{4}\right) a B_{1}-h \omega_{0}\left(1-\frac{3 a^{2}}{4}\right) a_{1}+\frac{\gamma h \omega_{0} a^{5}}{32 \omega_{0}^{2}}, \\
0= & 2 \omega_{0} a B_{1}-\frac{3 \gamma a^{2}}{2} a_{1}-A_{1} \frac{\partial A_{1}}{\partial a}+h\left(1-\frac{3 a^{2}}{4}\right) A_{1} \\
& +h \omega_{0} b_{1}\left(1-\frac{a^{2}}{4}\right)-\frac{3 \gamma^{2} a^{5}}{128 \omega_{0}^{2}}+\frac{h^{2} \omega_{0}^{2} a^{5}}{128 \omega_{0}^{2}} .
\end{aligned}
$$

The stationarity condition (1.7) for $i=2$ leads to the equation:

$$
A_{2}\left(a_{0}\right)=0 \quad \text { or } \quad h \omega_{0}\left(1-\frac{3 a_{0}^{2}}{4}\right) a_{1}+\frac{\gamma h \omega_{0} a_{0}^{5}}{32 \omega_{0}^{2}}=0
$$

from which we obtain:

$$
a_{1}=a_{10}=\frac{-\gamma}{2(1+3 \gamma)}
$$


The additional frequency $B_{1}\left(a_{0}\right)$ of order $\mu$ can be deduced from (2.15):

$$
B_{1}\left(a_{0}\right)=B_{10}=-\frac{9 \gamma^{2}+h^{2} \omega_{0}^{2}}{16 \omega_{0}^{3}} .
$$

Thus, the first two formulas for frequency are:

$$
\begin{aligned}
\omega_{I^{*}} & =\omega_{0}=\sqrt{1+3 \gamma} \\
\omega_{I I^{*}} & =\omega_{0}+\varepsilon B_{10}=\sqrt{1+3 \gamma}\left\{1-\frac{9 \gamma^{2}+h^{2}(1+3 \gamma)}{16(1+3 \gamma)^{2}}\right\} .
\end{aligned}
$$

In practice the stability condition in the first approximation is used and it is identical with the condition (1.2) given in section 1.

\section{Comparison and conclusion}

The formulas (1.20), (1.21) of $\omega_{I}, \omega_{I I}$ in section 1 and $(2.19),(2.20)$ of $\omega_{I *}, \omega_{I I *}$ in section 2 are identical with the formulas (2.17), (2.18) and (3.16), (3.17) obtained in [3] by the standard and the modified Poincaré method, respectively.

The degree of accuracy of these formulas has been shown in [3] and this allows us to affirm that the modified asymptotic procedure presented may be usefull.

It is not difficult-for instance-to affirm that the modified Poincaré method and the modified asymptotic method determine the same steady self-excited oscillations. Indeed, in [3] by using the dimensionless time $\tau=\omega t$ and by expanding

$$
x=x(\tau)=x_{0}(\tau)+\mu x_{1}(\tau)+\mu^{2} x_{2}(\tau)+\ldots
$$

following differential equations are obtained:

$$
\begin{aligned}
\omega_{0}^{2}\left(x_{0}^{\prime \prime}+x_{0}\right)= & 0 \\
\omega_{0}^{2}\left(x_{1}^{\prime \prime}+x_{1}\right)= & \left(\omega_{0}^{2}-1\right) x_{0}-\gamma x_{0}^{3}+h\left(1-x_{0}^{2}\right) \omega_{0} x_{0}^{\prime}, \\
\omega_{0}^{2}\left(x_{2}^{\prime \prime}+x_{2}\right)= & -2 \omega_{0} \omega_{1}\left(x_{1}^{\prime \prime}+x_{1}\right)+\left(\omega_{0}^{2}-1\right) x_{1}+2 \omega_{0} \omega_{1} x_{0}-3 \gamma x_{0}^{2} x_{1} \\
& +h\left(1-x_{0}^{2}\right)\left(\omega_{0} x_{1}^{\prime}+\omega_{1} x_{0}^{\prime}\right)-2 h x_{0} x_{1} \omega_{0} x_{0}^{\prime},
\end{aligned}
$$

where primes denote differentiation with respect to $\tau$.

The general solution of (3.1) satisfying the initial condition $x^{\prime}(0)=0$ is $x_{0}=a_{0} \cos \tau$. For steady self-excited oscillation $A_{1}\left(a_{0}\right)=0, A_{2}\left(a_{0}\right)=0$, So the difference between the equations (2.6), (2.13) and (3.2), (3.3) consists only in the difference between the notations $\left(\psi \rightarrow \tau, u_{1} \rightarrow x_{1}, u_{2} \rightarrow x_{2}, B_{1} \rightarrow \omega_{1}\right)$.

This publication is completed with the financial support from The Council for Natural Science of Vietnam.

\section{References}

1. Mitropolskii Yu. A., Nguyen Van Dao, Applied asymptotic method in nonlinear oscillations, Kluwer Academic Publishers, 1997.

2. Nguyen Van Dinh, On a variant of the asymptotic procedure (for weakly nonlinear autonomous systems), Vietnam Journal of Mechanics, NCST of Vietnam, 25 (2003). 
3. Nguyen Van Dinh and Tran Duong Tri, Frequency of a Van-Der-Pol oscillator with large cubic restoring nonlinearity, Vietnam Journal of Mechanics, NCST of Vietnam, 25 (2003).

Received June 1, 2003

\section{BIẾN THỂ CỦA PHUOONG PHÁP TIỆM CẬN CHO CHẤN TƯ VAN-DER-POL CÓ PHI TUYẾN HỒI PHỰC MẠNH BẬC BA}

Xét chấn từ Vanđécpôn có phi tuyến phục hồi mạnh bậc ba với tự kích yếu. Một biến thể của phương pháp tiệm cận được đề xuất và áp dụng. Kết quả thu được trùng với kết quả cho bởi một biến thể của phương pháp Poincaré với độ chính xác được nâng cao rõ rệt và có thể chấp nhận kể cả khi độ phi tuyến khá mạnh. 